\title{
Espacialização da umidade do solo por meio da temperatura da superfície e índice de vegetação
}

\author{
Helio L. Lopes ${ }^{1}$, Luciano J. de 0. Accioly², Flávio H. B. B. da Silva², \\ Maria do C. M. Sobral ${ }^{3}$, José C. de Araújo Filho $^{2} \&$ Ana L. B. Candeias ${ }^{3}$
}

RESU MO

0 estudo da umidade do solo é fundamental não só para a determinação da resiliência de ecossistemas e sua recuperação, mas também na modelagem da relação água-vegetação-atmosfera. $\mathrm{Na}$ aquisição dessas informações o sensoriamento remoto perfaz uma ferramenta importante e de potencial adequado para monitoramento e mapeamento. Visando à espacialização de índices relacionados à umidade, vários métodos têm sido propostos, embora sua aplicação ainda seja limitada. N este trabalho se aplicou o modelo de índice de umidade do solo (IU S) cujos objetivos foram: espacializar o IUS, estabelecer graus de desertificação, delimitar a área em processo de desertificação e verificar possíveis relações do IUS com parâmetros de água no solo. $\mathrm{Na}$ aplicação deste modelo se utilizaram, como dados de entrada, $\mathrm{O}$ NDVI (índice de vegetação da diferença normalizada) e a LST (temperatura da superfície) e se observou que o IUS representado pela média dos valores desses índices pode ser empregado na determinação do grau de degradação da superfície e para gerar classificação legendada, discriminando vários níveis de degradação ambiental. Constatou-se também que não houve relação direta do IUS com parâmetros físicos de retenção de umidade do solo. Desta forma, o sensoriamento remoto mostrou ser uma ferramenta significativa na avaliação de índices de umidade do solo em áreas degradadas tal como para delinear a dinâmica de borda em núcleo de desertificação.

Palavras-chave: índice de umidade do solo, NDVI, Seridó, desertificação

\section{Spatial distribution of soil moisture using land surface temperature and vegetation indices}

\begin{abstract}
A B STRACT
The study of soil moisture is important in determining the resilience of ecosystems and their recovery, as well as in the modeling of water-vegetation-atmosphere relationship. Remote sensing is an important tool for the acquisition, mapping and monitoring soil moisture through the surface temperature and vegetation indices. For the soil moisture content assessment, several methods have been proposed, how ever its application is still limited. In this work the soil moisture index (SMI) was applied and modeled with the objectives: to establish and delineate areas with different levels of desertification through SMI mapping and to map the dynamic of border, as well as to verify possible relationships betweem SMI and soil water parameters. In the application of this model as input data was used: NDVI (normal ized difference vegetation index) and LST (land surface temperature). It was observed that SM I accessed by the average of the SM I derived by NDVI and LST can be used in the determination of soil surface degradation and in the production of maps showing different levels of this degradation. It was also verified, that there was no direct relationship between SMI and physical parameters of soil moisture content. Remote sensing showed to be an important tool in the evaluation of soil moisture indices in degraded areas and to delineate the border effect in this desertification nucleus.
\end{abstract}

Key words: soil moisture index, NDVI, Seridó, desertification

${ }^{1}$ Colegiado de Engenharia Agronômica/U NIVASF. CEP 56304-205, Petrolina, PE. Fone (87) 3986-3802. Email: helio.lopes@univasf.edu.br Embrapa Solos/UEP. CEP 51020-240, Recife, PE. Fone (81) 3325-5988. Email: coelho@uep.cnps.embrapa.br Departamento de Engenharia Civil/UFPE. CEP 51000-000, Recife, PE. Fone (81)2126-8219. Email: msobral@ufpe.br 


\section{INTRODUÇÃO}

O conhecimento da umidade do solo é de fundamental importância devido ao papel ambiental relacionado a processos biológicos, ecológicos, hidrológicos e atmosféricos (Zhan et al., 2004; Ávila et al., 2010). Diversos pesquisadores vêm utilizando dados espectrais terrestres e orbitais para determinação espacial da umidade do solo (Zeng et al., 2004; Hassan et al., 2007; Kurc \& Benton 2010; Sanchez et al., 2010; Yichang et al., 2010). Vivoni et al. (2008) avaliaram, utilizando dados de sensoriamento remoto, a variabilidade espaçotemporal da umidade do solo em uma bacia hidrográfica, demonstrando sua variação em regiões complexas que possuem diferenças de vegetação, de propriedades do solo e de condições hidrológicas. Segundo Lopes et al. (2010), em trabalho realizado no bioma caatinga a temperatura da superfície varia com o uso e cobertura do solo. Essas variabilidades têm função relevante na variação de processos, como: precipitação e escoamento e na relação dos fluxos de calor latente e sensível (Eltahir, 1998).

De acordo com Zhan et al. (2004), variações na umidade do solo produzem mudanças significativas no balanço da energia da superfície. Desta forma, a umidade do solo é um parâmetro essencial na modelagem de processos da superfície e hidrológicos; sua determinação por dados de sensoriamento remoto é fundamental para a análise espacial em grandes áreas. O trabalho de Zhan et al. (2004) teve como objetivo avaliar o potencial do sensoriamento remoto no monitoramento de parâmetros biofísicos, como: LST, umidade do solo, emissividade, NDVI e outros parâmetros essenciais à análise de ecossistemas em bacias hidrográficas.

A umidade do solo tem grande efeito na magnitude da temperatura da superfície (LST) em virtude da sua marcante influência no armazenamento de energia térmica porém se ressalta que, em larga escala, a umidade do solo ainda não tenha sido bem estudada. Pesquisas nas quais se utilizaram espectro do visível, infravermelho termal e micro-ondas, têm mostrado potencial na estimativa das condições de umidade do solo (Vivoni et al., 2008; Baup et al., 2007; Das \& Mohanty, 2008; Katra et al., 2007).

Tem-se utilizado, em alguns trabalhos, a LST para determinar a umidade do solo (Zeng et al., 2010); outros investiram em modelar algoritmos para correlacionar a LST com a umidade do solo. Sun \& Pinker (2004), por exemplo, modelaram um novo algoritmo para determinar a LST a partir da umidade do solo.

Neste trabalho se modelam dados referentes à temperatura de superfície e índices de vegetação para espacializar informações sobre a umidade da superfície e sua relação com o estado da degradação ambiental cujo principal objetivo foi aplicar e adaptar o índice de umidade do solo (IUS), a um modelo empírico para determinação da umidade do solo, visando ao estudo de regiões degradadas da caatinga e com isto foi possível: espacializar o IUS, estabelecer graus de desertificação, delimitar a área em processo de desertificação, mapear o efeito de borda do núcleo de desertificação do Seridó e verificar possíveis relações do IUS com parâmetros de água no solo.

\section{Material E MÉTODOS}

\section{Área de estudo}

A região selecionada para o estudo abrange municípios situados entre os Estados da Paraíba e do Rio Grande do Norte (Figura 1). Aárea compreende o total de 31 municípios, em que 15 pertencem ao estado da Paraíba e 16 ao estado do Rio Grande do Norte. Este Núcleo de Desertificação tem altitude que varia de 200 a $700 \mathrm{~m}$ (Costa et al., 2001).

Um dos grandes impactos neste núcleo é o processo de desmatamento indiscriminado visando sobretudo à extração de lenha para a alimentação dos fornos das inúmeras olarias e cerâmicas instaladas na região. A vegetação da região é caracterizada como caatinga hiperxerófila na qual podem ser distinguidos, pelo menos, três padrões de densidade: caatinga densa, caatinga semidensa e caatinga aberta (Accioly et al., 2001). São característicos arbustos e árvores de até $2 \mathrm{~m}$ de altura em especial em áreas em que já ocorreu plantio de algodão e atualmente ocorre exploração da madeira. O estrato mais baixo é formado por pereiro (Aspidosperma pyrifolium), faveleira (Cnidoscolus quercifolius), catingueira (Poincianella pyramidalis) e vários cactus (Facheiroa squamosa, Pilosocereus gounellei), enquanto o estrato mais alto apresenta poucas umburanas (Myracrodruon urundeuva). Nas regiões mais degradadas ocorrem áreas com cobertura vegetal pouco densa, predominantemente de porte baixo e áreas abertas com formas e dimensões variadas, com presença de sulcos e voçorocas. Os dados climáticos são caracterizados por uma precipitação média anual de $497 \mathrm{~mm}$, velocidade dos ventos para uma altura de $10 \mathrm{~m}$ na faixa de $32,8 \mathrm{~m} \mathrm{~s}^{-1}$, podendo variar de $2,5 \mathrm{a} 46 \mathrm{~m} \mathrm{~s}^{-1}$, e a temperatura de 20 a $32{ }^{\circ} \mathrm{C}$.

Os solos dominantes na região, conforme o Sistema Brasileiro de Classificação do Solo (EMBRAPA, 2006), pertencem às classes dos Luvissolos Crômicos (TC), Planossolos Nátricos (SN), Neossolos Litólicos (RL); Neossolos Regolíticos (RR) e em baixas proporções, a dos Argissolos Vermelho-Amarelos (PVA), Argissolos Vermelhos (PV), Argissolos Amarelos (PA), Cambissolos Háplicos (CX) e Neossolos Flúvicos (RY). Onde ocorrem os Luvissolos e solos associados tem-se especialmente um nível de erosão laminar acentuado e, em sulcos, esses últimos, em diversas profundidades. A Figura 2 mostra o arranjamento espacial das principais classes de solo para uma área do núcleo que compreende cinco municípios.

Obtiveram-se, também, informações sobre retenção de umidade e disponibilidade hídrica de solos de uma área da região do Seridó, para posterior comparação com o índice de umidade do solo e verificação de correlação entre os parâmetros. As análises foram realizadas segundo metodologias da Embrapa (EMBRAPA, 1997).

\section{Processamento dos dados}

Para a modelagem do índice de umidade do solo - IUS, utilizaram-se imagens LANDSAT-TM5 do ano de 2002, referentes à estação seca. As órbitas-ponto foram utilizadas: 215/64, 215/65, 216/64 e 216/65 e realizado um recorte para a área de estudo. 


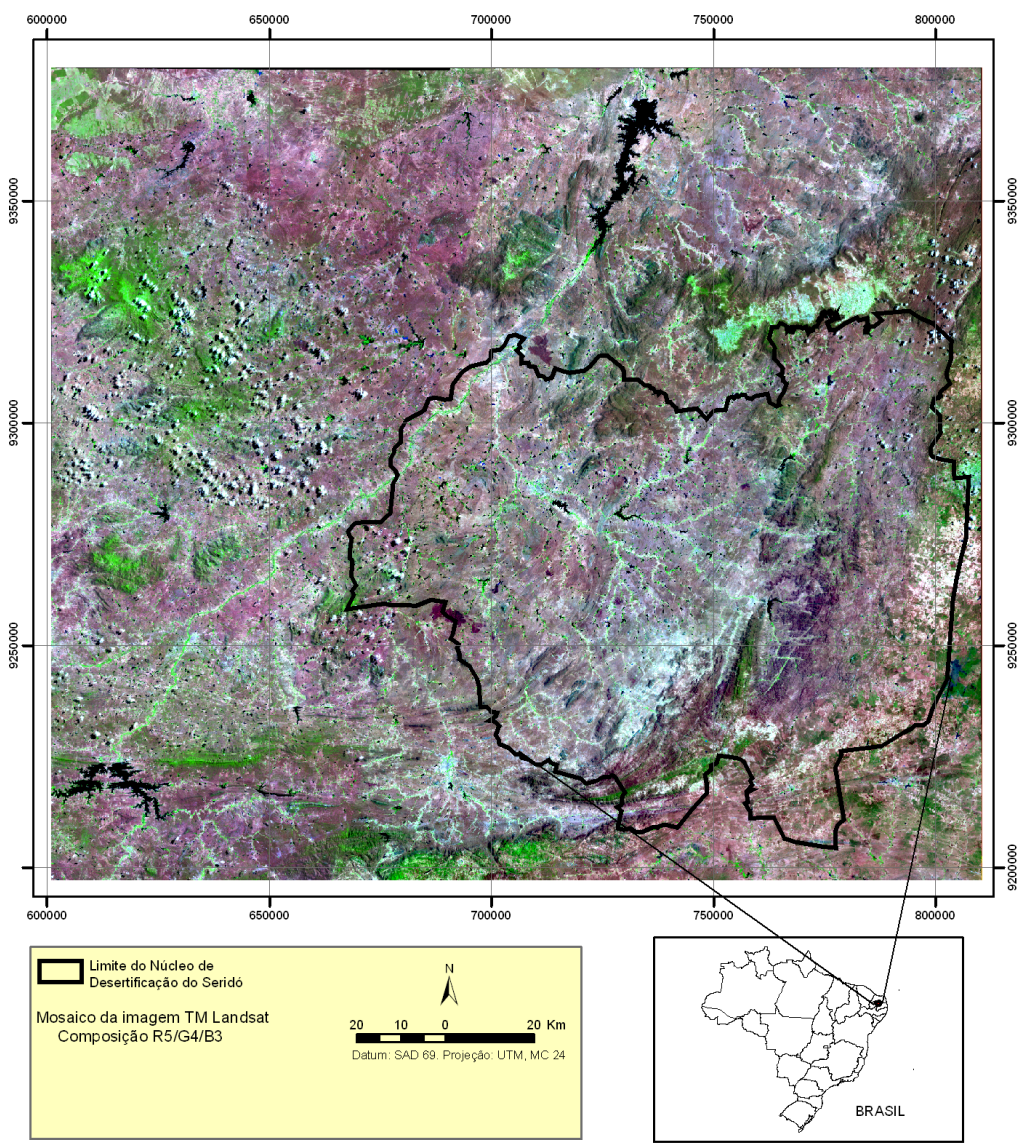

Figura 1. Localização geográfica do núcleo de desertificação do Seridó
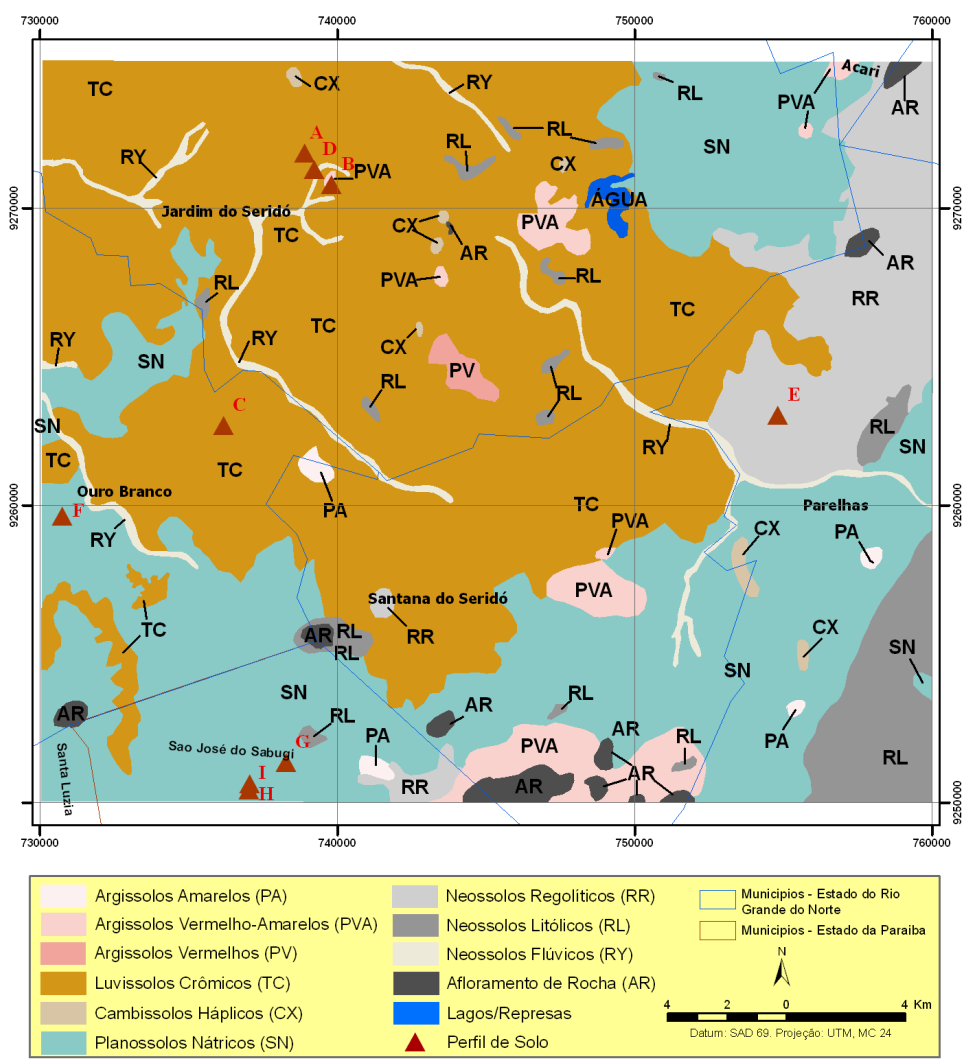

Figura 2. Mapa em que são destacadas as subordens de solos dominantes nos municípios de Parel has, O uro Branco, Jardim do Seridó, Santana do Seridó, no estado do Rio G rande do N orte, e São José do Sabugi, no Estado da Paraíba. Mapa adaptado de Silva et al. (2002) 
Todas as imagens foram registradas no sistema de referência SAD-69, projeção UTM, utilizando-se um polinômio de segunda ordem e amostragem, pelo método de vizinho mais próximo. A precisão no registro foi da ordem do subpixel. A correção atmosférica seguiu o método do histograma (pixel mais escuro). Após se efetuar a correção realizou-se a conversão dos níveis de cinza (NC) das imagens para eflectância. O trabalho foi realizado em duas etapas, em que na primeira os valores de NC foram convertidos à radiância espectral na banda $\mathrm{i}\left(\mathrm{L}_{\mathrm{i}} \mathrm{em} \mathrm{W} \mathrm{m} \mathrm{m}^{-2} \mathrm{sr}^{-1} \mathrm{~m}^{-1}\right)$ através dos valores de radiância mínima $\left(\mathrm{L}_{\text {imin }}\right)$ e máxima $\left(\mathrm{L}_{\text {imax }}\right)$ para a banda considerada, obtidos a partir da calibração absoluta de pós-lançamento do sensor Landsat 7 ETM+. A Eq. 1 converte os valores de NC em radiância (Allen et al., 2002):

$$
\mathrm{L}_{\mathrm{i}}=\left(\frac{\mathrm{L}_{\mathrm{imax}}-\mathrm{L}_{\mathrm{imin}}}{255}\right) \times \mathrm{NC}_{\mathrm{i}}
$$

em que:

$\mathrm{NC}_{\mathrm{i}}$ - Nível de cinza na banda $\mathrm{i}$

Valores de $\mathrm{L}_{\text {imin }}$ e de $\mathrm{L}_{\text {imax }}$ para as imagens do Landsat 7 ETM+ foram extraídos do Handbook do Landsat 7.

Uma vez convertidos os valores de $\mathrm{NC}$ em radiância, a próxima etapa foi a remoção do efeito de primeira ordem da atmosfera, para o que se utilizaram os valores mínimos de radiância, dados por alvos escuros. Os valores de L para esses alvos podem ser atribuídos ao somatório do sinal de ruído do instrumento com a fração da energia refletida pela atmosfera, que atinge diretamente o sensor para a banda considerada ("path radiance").

A conversão de radiância para reflectância é dada pela Eq. 2 (Allen et al., 2002):

$$
\rho_{\mathrm{i}}=\frac{\pi \times \mathrm{L}_{\mathrm{ic}} \times \mathrm{d}^{2}}{\operatorname{Esun}_{\mathrm{i}} \times \cos ^{2} \theta}
$$

em que:

$\mathrm{L}_{\mathrm{ic}}$ - radiância corrigida na banda $\mathrm{i}, \mathrm{W} \mathrm{m}^{-2} \mathrm{sr}^{-1} \mathrm{~m}^{-1}$

d - distância Sol-Terra, unidade astronômica

Esun $_{\mathrm{i}}$ - irradiância solar no topo terrestre $\left(\mathrm{W} \mathrm{m}^{-2} \mathrm{~m}^{-1}\right)$ para a banda $\mathrm{i}$

$\theta \quad$ - ângulo solar zenital

Após a determinação da reflectância para cada imagem realizou-se a espacialização do NDVI, de acordo com a Eq. 3:

$$
\mathrm{NDVI}=\frac{\rho_{\mathrm{NIR}}-\rho_{\mathrm{RED}}}{\rho_{\mathrm{NIR}}+\rho_{\mathrm{RED}}}
$$

em que:

$\rho_{\mathrm{NIR}}$ - reflectância no infravermelho

$\rho_{\mathrm{RED}}$ - reflectância no vermelho

Para modelagem da LST calculou-se a radiância na banda do termal e sua espacialização foi efetivada pela Eq. 4, seguinte (Bastiaanssen \& Bakker, 2000):

$$
\mathrm{LST}=\frac{1260}{\ln \left(\frac{61,6}{\mathrm{~L}_{\mathrm{TM} 6}}+1\right)} / \varepsilon^{0,25}
$$

em que:

$\mathrm{L}_{\mathrm{TM} 6}$ - radiância na banda termal;

$\varepsilon \quad$ - emissividade

A partir da determinação da temperatura da superfície em radiância aplicou-se a equação do índice de umidade do solo (IUS). O índice IUS tem valores de 0 , para superfícies mais secas, e 1, para superfícies mais úmidas (Zhan et al., 2004). O IUS foi calculado diretamente da temperatura da superfície pela Eq. 5:

$$
\mathrm{IUS}_{\mathrm{LST}}=\frac{\mathrm{LST}_{\max }-\mathrm{LST}}{\mathrm{LST}_{\max }-\mathrm{LST}_{\min }}
$$

donde:

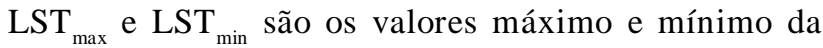
temperatura da superfície para a região de estudo. LST é determinada pela Eq. 4, para cada pixel.

O IUS também foi derivado do índice de vegetação (NDVI) adaptado da equação do IUS e derivado da temperatura da superfície.

A equação do IUS derivado do índice de vegetação (NDVI) foi modelada por meio da Eq. 6:

$$
\mathrm{IUS}_{\mathrm{NDVI}}=1-\left(\frac{\mathrm{NDVI}_{\mathrm{max}}-\mathrm{NDVI}}{\mathrm{NDVI}_{\max }-\mathrm{NDVI}_{\min }}\right)
$$

A constante 1 refere-se à inversão dos valores pois, quanto maior o valor do NDVI maior também o índice de umidade da superfície (Wang et al., 2010).

$\mathrm{O} \mathrm{NDVI}_{\max }$ e o NDVI ${ }_{\text {min }}$ se referem aos valores de máximo e mínimo de NDVI que ocorram na região de estudo. O NDVI na Eq. 6 é o valor calculado em cada pixel.

Neste estudo se utilizou o IUS médio gerado a partir do

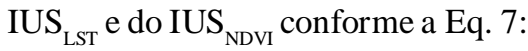

$$
\mathrm{IUS}=\frac{\mathrm{IUS}_{\mathrm{LST}}+\mathrm{IUS}_{\mathrm{NDVI}}}{2}
$$

sendo:

IUS $_{\text {LST }}$ o índice de umidade do solo derivado da temperatura da superfície e IUS $_{\mathrm{NDVI}}$ o índice de umidade do solo derivado do índice de vegetação (NDVI).

Após a determinação das imagens do NDVI e LST realizouse o mosaico das mesmas para se determinar os IUS ${ }_{\mathrm{LST}}$ e IUS $_{\mathrm{NDVI}}$ e o IUS final. 


\section{RESULTADOS E DISCUSSÃO}

Na Figura 3 se tem a imagem do IUS médio do solo. Percebese que no perímetro que delimita a região do Seridó existem áreas nas quais domina o IUS menor que 0,15 . Nesta região a degradação da caatinga é intensa, com existência de diversas formas de erosão laminar e em sulcos. Em geral, o IUS variou de 0 para as áreas desertificadas e com queimadas, até 0,57 para áreas com vegetação densa. Yichang et al. (2010) mapearam valores de umidade do solo variando de 0 a 0,21 em uma área com a maior parte da cobertura vegetativa variando de 27 a $36 \%$.

Além dos municípios pertencentes à região do núcleo de desertificação do Seridó, também se evidencia a degradação em outros municípios circunvizinhos do Rio Grande do Norte e da Paraíba, corroborando com resultados de Candido et al. (2002). Por conseguinte, os resultados sugerem que seria fundamental observar a evolução da degradação na região, conforme estudo realizado por Wang et al. (2010) que verificaram o impacto da mudança do uso e cobertura do solo na umidade em uma bacia hidrográfica, através do modelo TemperaturaVegetação utilizando 34 pontos de validação. Esta análise seria sugerida em relação ao avanço da desertificação, particularmente por meio da dinâmica de borda referente aos parâmetros biofísicos modelados e se verificando sua influência no avanço do risco à desertificação, através de comparações espaço-temporal, conforme metodologia aplicada por Lopes et al. (2009). Por meio deste estudo se verificam áreas degradadas no município de Patos e circunvizinhos e no município de Santana dos Matos.
Para um pixel localizado na região, conforme Figura 4A, o valor do IUS foi igual a 0,123 , numa área em que os solos se encontram bastante erodidos, de modo que já se percebe a exposição do substrato rochoso.

Obteve-se, para um pixel correspondente à vegetação tipo caatinga hiperxerófila (savana estépica arborizada) e conforme visto na Figura 4B, o valor do IUS de 0,403, ocorrido no município de Equador, RN.

Este valor revela a influência da vegetação nas condições de umidade do solo e sua relação direta com o IUS. Resultados semelhantes obtiveram Wang et al. (2010) ao utilizar oNDVI eLST para estimar a umidade do solo. Apesar da relação é notória a necessidade de calibração do modelo com dados de campo e posteriores análises estatísticas para verificar coeficientes de determinação, além de possibilidades de se relacionar o modelo com a paisagem, particularmente com os tipos de soloexistentes na região.

Os resultados evidenciam que, entre os municípios da região do Seridó, os mais atingidos pela degradação são: São Mamede, Várzea, Santa Luzia, Juazeirinho, Seridó, Cubati e São José do Sabugi, pertencentes ao estado da Paraíba, e Ipueira, São João do Sabugi, Ouro Branco, Jardim do Seridó, Jardim de Piranhas, Santana do Seridó, São Fernando, Parelhas, São José do Seridó e Caicó, pertencentes ao estado do Rio Grande do Norte. Percebe-se, pela Figura 3, que os municípios de Patos, Quixaba, São José do Bonfim e Santo André se encontram com uma área do município comprometida com a degradação, tal como os municípios de Santana dos Matos, São Rafael, Itajá, Paraú e Campo Grande, pertencentes aos estados da Paraíba e Rio Grande do Norte, respectivamente.

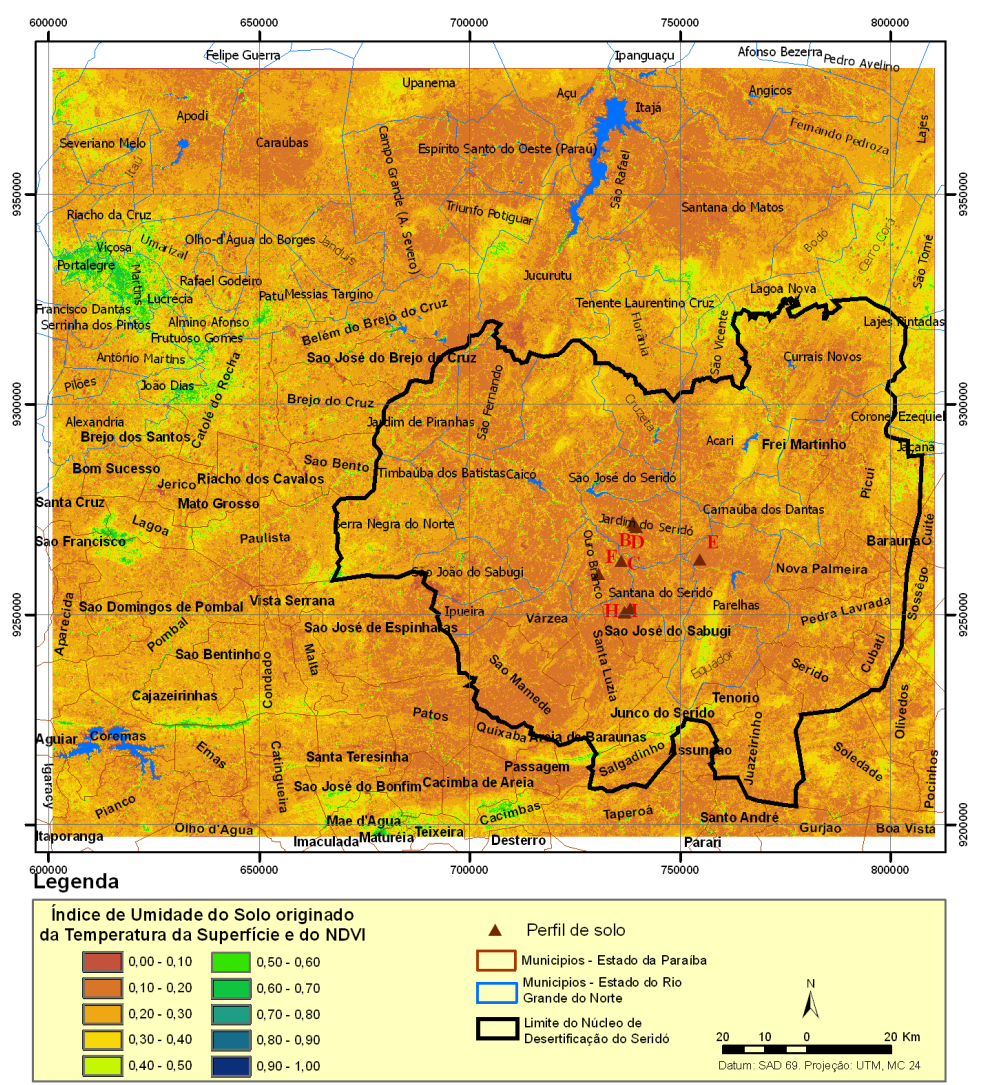

Figura 3. Mapa do índice de umidade do solo (IU S) para o núcleo de desertificação do Seridó (Paraíba e Rio G rande do Norte) 
A.

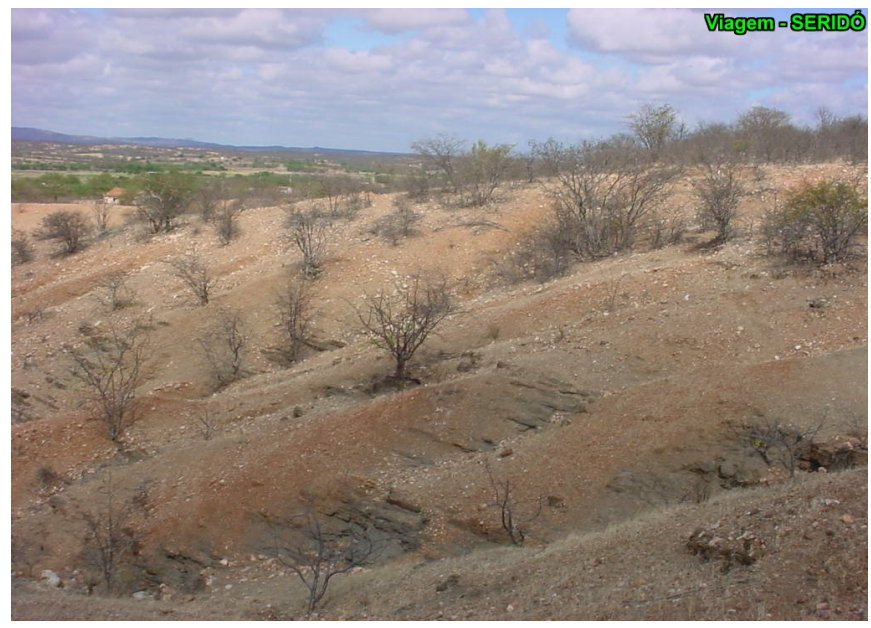

B.

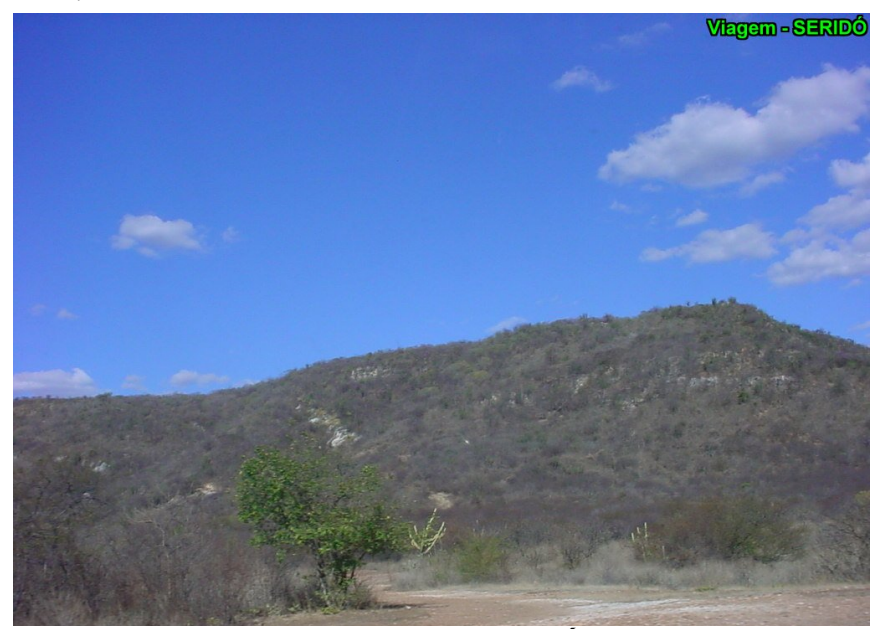

Figura 4. Paisagens da região. A) Área degradada no município de Santana do Seridó. B) Foto da caatinga hiperxerófila (savana estépica arborizada) no M unicípio de Equador

Na região do Seridó não somente as condições naturais favorecem a degradação ambiental (clima e solos) mas, particularmente, as atividades antrópicas vigentes na região. Nos citados municípios ocorre devastação da caatinga para utilização da lenha em olarias e atividades de mineração além de ter havido, no passado, o cultivo da cultura do algodão que não oferecia proteção aos solos. Em consequência, as causas naturais somadas às antrópicas têm levado à degradação ambiental, particularmente a erosão dos solos, conforme visto na Figura 4A. Destaca-se que em áreas expressivas da região do Seridó a vegetação natural já não consegue se recompor, permanecendo com porte pequeno e de forma raleada, deixando áreas expressivas da superfície do solo expostas aos processos erosivos.

A partir da espacialização do IUS pode-se comparar os valores do índice com os dados físicos dos solos, a partir de amostras coletadas no ano de 2001. Os dados comparados com o IUS foram: água retida a 1,5 MPa, 0,003 MPa e água disponível referentes a nove perfis de solo (Tabela 1). Segundo Mello et al. (2005), algumas variáveis, como a quantidade de areia, de argila e de matéria orgânica, influenciam na umidade do solo e também na resposta espectral da superfície (Ben-Dor et al., 2002; Selige et al., 2006; Lagacherie et al., 2008). Entretanto, a maior variação na umidade do solo ocorre entre 0 e $5 \mathrm{~cm}$ (Dallacort et al., 2010), que ainda observaram que os coeficientes de variação na camada de 0 a $20 \mathrm{~cm}$ são sempre superiores aos da camada subsequente, conforme resultados de Araújo et al. (2001). Wang et al. (2007) realizaram correlações lineares bivariadas entre o NDVI e o conteúdo volumétrico de água no solo, encontrando coeficiente de correlação entre 0,51 a 0,84 . A Figura 5 mostra as relações dos parâmetros físicos com o IUS.

Procurou-se mostrar, nos gráficos a seguir, possíveis relações do IUS com parâmetros de retenção de água no solo.

Verifica-se, de acordo com a Figura 5, que o índice de umidade do solo não tem qualquer relação direta com a água retida a 1,5 MPa, 0,003 MPa e água útil, conforme sugerem os coeficientes $\left(\mathrm{R}^{2}\right)$ praticamente nulos; desta forma, o conhecimento de um parâmetro nada contribui para a previsão de outro e, portanto, o IUS poderá apenas ter relação com respostas da superfície referentes à reflectância em relação ao NDVI e emitância quanto à temperatura da superfície, conforme Figura 6 , e com o conteúdo volumétrico de água no solo, conforme concluíram Wang et al. (2007).

De acordo como a Figura 6, observa-se boa correlação do índice de umidade médio com os parâmetros NDVI e LST com coeficiente determinístico de 0,90 e 0,87 , respectivamente. Esta relação mostra uma medida significativa da intensidade de relação entre os parâmetros, denotando uma relação de causa e efeito entre os parâmetros, em função das mudanças da superfície.

Tabela 1. Dados de retenção de umidade e disponibilidade hídrica referentes a nove perfis de solo da região do Seridó e valores do IUS

\begin{tabular}{ccccccc}
\hline Perfil & Horizonte & $\mathbf{1 , 5} \mathbf{M P a}$ & $\mathbf{0 , 0 0 3} \mathbf{M P a}$ & Água Útil & Data & IUS \\
A & $\mathrm{A}$ & 5,1 & 9,4 & 4,3 & $11 / 10 / 2001$ & 0,165 \\
B & A & 5,4 & 11,0 & 5,6 & $11 / 10 / 2001$ & 0,199 \\
C & Bt & 5,4 & 10,4 & 5,0 & $11 / 10 / 2001$ & 0,123 \\
D & A & 5,5 & 12,4 & 6,9 & $11 / 10 / 2001$ & 0,165 \\
E & A & 2,7 & 6,2 & 3,5 & $10 / 10 / 2001$ & 0,129 \\
F & A & 6,3 & 13,1 & 6,8 & $11 / 10 / 2001$ & 0,159 \\
G & A & 1,2 & 2,6 & 1,4 & $11 / 10 / 2001$ & 0,185 \\
H & A & 2,2 & 5,8 & 3,6 & $10 / 10 / 2001$ & 0,143 \\
I & A1 & 2,5 & 5,5 & 3,0 & $11 / 10 / 2001$ & 0,151 \\
\hline
\end{tabular}


A.

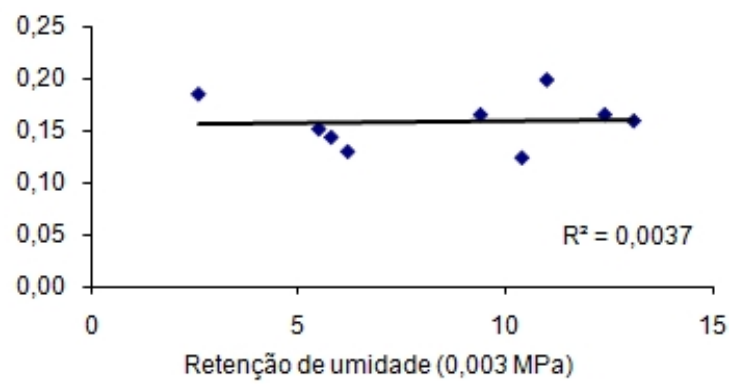

B.

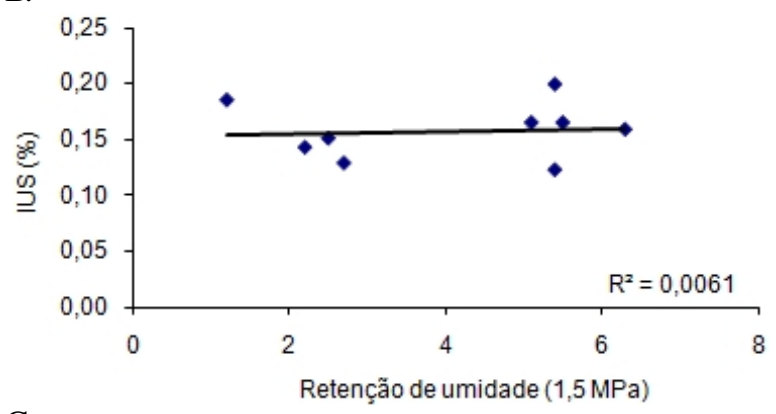

C.

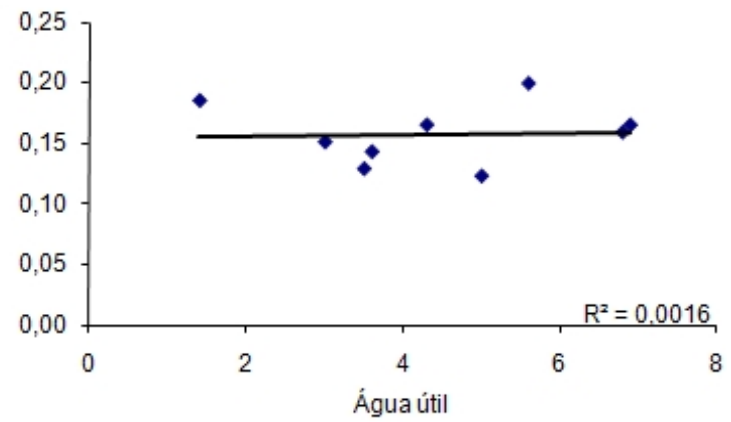

Figura 5. Relação entre o IU S e os parametros água retida. A) a 0,003 M Pa; B) a 1,5 M Pa; e C) água disponível

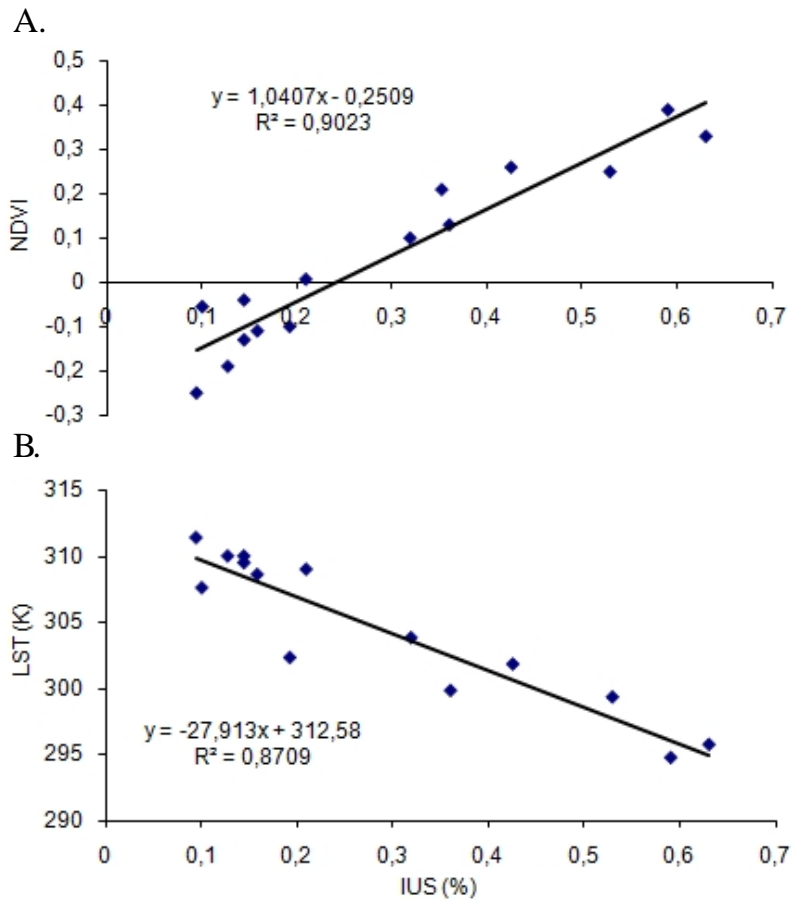

Figura 6. Relação entre o IU S e os parâmetros: A) NDVI e B) LST

\section{CONCLUSÕES}

1. O índice de umidade do solo variou com a paisagem indicando uma relação direta com a densidade de vegetação relacionada com o NDVI.

2. Verifica-se que ocorre uma degradação intensa nos municípios vizinhos ao Núcleo, resultando no aumento da área da região de desertificação do Seridó.

3. O índice de umidade do solo adaptado neste trabalho, não possui relação direta com a retenção de umidade a 1,5 $\mathrm{MPa}, 0,003 \mathrm{MPa}$ e água útil, mas apenas com características da paisagem.

4. O sensoriamento perfaz uma ferramenta que pode determinar índices importantes para avaliar áreas em processo de degradação e sua quantificação.

\section{LITERATURA CITADA}

Accioly, L. J. de O.; Oliveira, M. J. A. de; Silva, F. H. B. B. da; Avaliação de Mudanças no Albedo do Núcleo de Desertificação do Seridó através de Imangens Landsat TM. In: congresso Brasileiro de Sensoriamento Remoto, 10, 2001, Foz do Iguaçu. Anais do X Simpósio Brasileiro de Sensoriamento Remoto, São José dos Campos: INPE, 2001, p. 549-556, 2001.

Allen, R. G.; Tasumi, M.; Trezza, R.; Waters, R.; Bastiaanssen, W. SEBAL (Surface Energy Balance Algorithms for Land). Advance Training and Users Manual - Idaho Implementation, version 1.0. Idaho: University of Idaho, 2002.97p.

Araújo, E. C. B. de; Aguiar, J. V. de; Costa, R. N. T. Calibração de um modelo de umidade para um solo aluvial sem cobertura vegetal. Revista Brasileira de Engenharia Agrícola e Ambiental, v.5, p.444-449, 2001.

Ávila, L. F.; Mello, C. R. de; Silva, A. M. da. Continuidade e distribuição espacial da umidade do solo em Bacia Hidrográfica da Serra da Mantiqueira. Revista Brasileira de Engenharia Agrícola e Ambiental, v.14, p.1257-1266, 2010.

Bastiaanssen, W.; Bakker, M. Use of satellite data in agricultural water management. Embrapa Semiárido. Petrolina: 2000. 81p.

Baup, F.; Mougin, E.; Rosnay, P. de; Timouk, F.; Chênerie, I. Surface soil moisture estimation over the AMMA Sahelian site in Mali using ENVISAT/ASAR data. Remote Sensing of Environment, v.109, p.473-481, 2007.

Ben-Dor, E.; Patkin, K.; Banin, A.; Karnieli, A. Mapping of several soil properties using DAIS-7915 hyperspectral scanner data-a case study over clayey soils in Israel. International Journal of Remote Sensing, v.23, p.1043-1062, 2002.

Candido, H. G.; Barbosa, M. P.; Silva, M. J. da. Avaliação da degradação ambiental de parte do Seridó Paraibano. Revista Brasileira de Engenharia Agrícola e Ambiental, v.6, p.368371, 2002. 
Costa, T. C. e C. da; Accioly, L. J. de O.; Oliveira, M. A. J. de; Burgos, N.; Silva, F. H. B. B. da. Mapeamento da fitomassa da caatinga do núcleo de desertificação do Seridó, Pelo índice de área de planta (IAP) e o índice de vegetação da diferença Normalizada (NDVI), obtido com dados do sensor landsat 7 TM. In: congresso Brasileiro de Sensoriamento Remoto, 10, 2001, Foz do Iguaçu. Anais do X Simpósio Brasileiro de Sensoriamento Remoto, São José dos Campos: INPE, 2001, p.1563-1573.

Das, N. N.; Mohanty, B. P.; Temporal dynamics of PSR-based soil moisture across spatial scales in an agricultural landscape during SMEX02: A wavelet approach. Remote Sensing of Environment, v.112, p.522-534, 2008.

Dallacort, R.; Freitas, P. S. L. de; Faria, R. T. de; Gonçalves, A. C. A.; Jácome, A. G.; Rezende, R. Soil water balance simulated by CROPGRO-Drybean model for edaphoclimatic conditions in Maringá Revista Brasileira de Engenharia Agrícola e Ambiental, v.14, p.351-357, 2010.

Eltahir, E. A. B. A soil moisture rainfall feedback mechanism 1. Theory and observations. Water Resources Research, v.34, p.765-776, 1998.

EMBRAPA - Empresa Brasileira de Pesquisa Agropecuária. Centro Nacional de Pesquisa de Solos (Rio de Janeiro, RJ). Manual de métodos de análise de solos. 2.ed. rev. atual. Rio de Janeiro: Embrapa Solos, 1997. 212p.

EMBRAPA - Empresa Brasileira de Pesquisa Agropecuária. Centro Nacional de Pesquisa de Solos (Rio de Janeiro, RJ). Sistema brasileiro de classificação de solos. 2.ed. Rio de Janeiro: Embrapa Solos, 2006. 306p.

Hassan, Q. K.; Bourque, C. P. A.; Meng, F. R.; Cox, R. M. A wetness index using terrain-corrected surface temperature and normalized difference vegetation index derived from Standard MODIS Products: An evaluation of its use in a humid forest-dominated region of eastern Canada. Sensors, v.7, p.2028-2048, 2007.

Katra, I.; Blumberg, D. G.; Lavee, H.; Sarah, P. Topsoil moisture patterns on arid hillsides - Micro-scale mapping by thermal infrared images. Journal of Hydrology, v.334, p.359-367. 2007.

Kurc, S.A.; Benton, L.M. Digital image-derived greenness links deep soil moisture to carbon uptake in a creosotebushdominated shrubland. Journal of Arid Environments, v.74, p.585-594, 2010.

Lagacherie, P.; Baret, F.; Feret, J. B.; Madeira Netto, J.; RobbezMasson, J. M. Estimation of soil clay and calcium carbonate using laboratory, field and airborne hyperspectral measurements. Remote Sensing of Environment, v.112, p.825835. 2008.

Lopes, H. L.; Candeias, A. L. B.; Accioly, L. J. de O.; Sobral, M. do C. M. Modelagem de parâmetros biofísicos para desenvolvimento de algoritmo para avaliação e espacialização de risco a desertificação. Boletim de Ciências Geodésicas, v.15, p.652-668, 2009.
Lopes, H. L.; Candeias, A. L. B.; Accioly, L. J. O.; Sobral, M. do C. M.; Pacheco, A. P. Parâmetros biofísicos na detecção de mudanças na cobertura e uso do solo em bacias hidrográficas. Revista Brasileira de Engenharia Agrícola e Ambiental, v.14, p.1210-1219, 2010.

Mello, C. R. de; Oliveira, G. C. de; Ferreira, D. F.; Lima, J. M. de; Lopes, D. Modelos para determinação dos parâmetros da equação de van Genuchten para um Cambissolo. Revista Brasileira de Engenharia Agrícolae Ambiental, v.9, p.23-29, 2005.

Sánchez, N.; Martínez-Fernández, J.; Calera, A.; Torres, E.; Pérez-Gutiérrez, C. Combining remote sensing and in situ soil moisture data for the application and validation of a distributed water balance model (HIDROMORE). Agricultural Water Management, v.98, p.69-78, 2010.

Selige, T.; Böhner, J.; Schmidhalter, U. High resolution topsoil mapping using hyperspectral image and field data in multivariate regression modelling procedures. Geoderma, v.136, p.235-244, 2006.

Silva, F. H. B. B da.; Burgos, N.; Accioly, L. J. de O.; Costa, T. C. C. da; Oliveira, M. A. J. de. Caracterização dos recursos naturais de uma área piloto do núcleo de desertificação do Seridó, Estados do Rio Grande do Norte e Paraíba - Escala 1:100.000. Rio de Janeiro: Embrapa Solos, 2002. 55p. Boletim de Pesquisa 04

Sun, D.; Pinker, R. T. Case study of soil moisture effect on land surface temperature retrieval. IEEE Geoscience and Remote Sensing Letters, v 1, p.127-130, 2004.

Vivoni, E. R.; Gebremichael, M.; Watts, C. J.; Bindlish, R.; Jackson, T. J. Comparison of ground-based and remotely sensed surface soil moisture estimates over complex terrain during SMEX04. Remote Sensing of Environment. v.112, p.314-325, 2008.

Wang, H.; Li, X.; Long, H.; Xu, X.; Bao, Y. Monitoring the effects of land use and cover type changes on soil moisture using remote-sensing data: A case study in China's Yongding River basin. Catena, v.82, p.135-145, 2010.

Wang, X.; Xie, H.; Guan, H.; Zhou, X. Different responses of MODIS-derived NDVI to root-zone soil moisture in semiarid and humid regions. Journal of Hydrology, v.340, p.1224, 2007.

Yichang, W.; Fang, Z.; Liping, Z.; Lingling, K.; Xiaoqiang, L. Estimating soil moisture in semi-arid region by remote sensing based on TM data. IEEE Transactions on Geosciences and Remote Sensing, v.1, p.1-5, 2010.

Zeng, Y.; Feng, Z.; Xianga, N. Assessment of soil moisture using Landsat ETM+ temperature/vegetation index in semiarid environment. IEEE Transactions on Geosciences and Remote Sensing, v.6, p.4036-4039, 2004.

Zhan, Z.; Qin, Q.; Wang, X. The application of LST/NDVI index for monitoring land surface moisture in semiarid area. IEEE Transactions on geosciences and Remote Sensing, v.3, p.1551-1554, 2004. 